\title{
Characterization of carbapenem non-susceptible Gram-negative Bacilli isolated from the feces of 10,000 inpatients in Southern China
}

\author{
Xiaonv Duan \\ Southern Medical University \\ Si Li \\ Southern Medical University \\ Yuan Peng \\ Southern Medical Univeristy \\ YongYu Rui ( $\nabla$ yongyuruigroup@sina.com) \\ Southern Medical University
}

\author{
Research \\ Keywords: fecal carriage, carbapenem, gram-negative bacilli, colistin \\ Posted Date: August 25th, 2020 \\ DOI: https://doi.org/10.21203/rs.3.rs-53371/v1 \\ License: (c) (7) This work is licensed under a Creative Commons Attribution 4.0 International License. Read Full License
}




\section{Abstract \\ Background}

Carbapenem non-susceptible Gram-negative bacilli (CNS-GNB) were dominant pathogen causing clinical infections. The human intestine was important reservoir of GNB, but there were few studies to analysis the prevalence of fecal colonization with them.

\section{Methods}

Fecal samples were collected from hosiptal screening test for GNB was conducted by using home-made MacConkey agar. Antimicrobial susceptibility was determined by the automatic microbiology analyzer and drug-resistant genes were characterized by polymerase chain reaction assays and DNA sequencing. The whole genome sequencing were used to analysis the characteristic of genetic structure of the isolates.

\section{Results}

A total of 680 CNS-GNB were collected. Acinetobacter spp. were the dominant species (33.8\%) of the 22 genera. Carbapenemase genes were identified in 307 isolates (45.1\%), including 206 (30.3\%) bla ${ }_{N D M} ; 1$ (7.5\%) bla VIM-2 $_{2} 48$ (7.1\%) bla $a_{M P}$, and seven (1.0\%) bla $a_{K P C-2}$. The bla ${ }_{N D M}$ genes were first detected in three isolates, Providencia vermicola, Achromobacter spp., and Cupriavidus gilardii. Co-existence of bla ${ }_{V I M}$ and $b / a_{I M P}$ genes was detected in five isolates; Achromobacter co-producing VIM and IMP has not been previously reported. The $m c r-1$ gene was identified in five strains of $A c i n e t o b a c t e r$ and one strain of $K$. pneumoniae. In addition, we detected seven isolates harboring the $b / a_{A F M-1}$ gene, a novel metallo- $\beta$-lactamase gene. This was first genomic analysis of ST11 K. pneumoniae co-producing NDM-5 and mcr-1, which revealed that blaNDM-5 and mcr-1 are located on two different plasmids. The plasmid harboring blaNDM-5, which was composed of a typical IncX3-type backbone, and the mcr-1 gene, was located between an IS30-like element ISApl1 and a PAP2-like encoding gene in the IncHI2-type plasmid.

\section{Conclusions}

the overall prevalence of fecal carriage of CNS-GNB in 10,000 stool samples was 7.45\%, and more than half of CNS-GNB produced carbapenemase. Most CNS-GNB cases were associated with infectious disease, multiple hospitalizations, or long-term care, and a high prevalence of underlying disease.

\section{Introduction}

Gram-negative Bacilli (GNB) are implicated in a wide range of diseases, such as pneumonia, meningitis, septicemia, and urinary tract infections [1]. According to China Antimicrobial Resistance Surveillance System (CARSS) monitoring data (http://www.carss.cn/), GNB have been shown to account for 70.6\% of the total number of bacteria in hospitals, which are resistant to most available antibiotics and have developed built-in abilities to acquire new ways of resistance [2]. Carbapenem antibiotics have been reserved as drugs of last resort for salvage treatment of infections caused by multidrug-resistant Gram-negative bacteria, but the emergence of carbapenem-resistant GNB (CR-GNB) poses a global healthcare challenge because therapeutic options are limited. CR-GNB represent difficult-to-treat infections in hospitalized patients and are associated with high mortality [3]. In recent years, the resistance to carbapenem of GNB has spread rapidly, which has led to increased CR-GNB, and asymptomatically colonized patients might act as important reservoirs for transmission.

The main carbapenem-resistance mechanism of GNB is the production of carbapenemases capable of hydrolyzing carbapenems; these enzymes have been characterized into various classes, including Ambler class A $\beta$-lactamases, such as KPC and GES; Ambler class B metallo- $\beta$-lactamases, such as IMP, VIM, and NDM $[4,5]$; and Ambler class D oxacillinases (OXAs). Carbapenemase genes are generally located on plasmids, which can disseminate resistance horizontally by mobile genetic elements or mobile plasmids.

Colistin has been used as an effective clinical therapeutic against carbapenem-resistant bacteria. However, colistin resistance poses a substantial public health risk because it further limits treatment options in patients with infections caused by multidrug-resistant Gram-negative bacteria, including CR-GNB. The recent discovery of transferable plasmid-mediated colistin resistance genes between bacteria has further increased the risk of spread of colistin resistance [6]. The $\mathrm{mcr}$ gene has been linked to colistin resistance and can be transferred via plasmids [7]. Strains that are not sensitive to carbapenems and show resistance to colistin can significantly increase the risk of death from clinical infection, so it is necessary to detect the presence of colistin resistance genes.

Few studies have described the prevalence of fecal colonization with carbapenem non-susceptible GNB in China. In the present study, we collected 680 carbapenem non-susceptible Gram-negative bacilli (CNS-GNB) isolated from fecal survey samples of 10,000 patients and studied the drug-resistant and transmission mechanism by analyzing resistance genes and the genomic structure.

\section{Materials And Methods Patients and specimens}

All samples were obtained randomly and were not selected for on the basis of suspected enteric infection or diarrhea. A total of 10,000 fecal survey samples from in-patients who underwent routine stool examinations on the first day of hospitalization were prospectively and consecutively collected from July 2013 to June 2015 at Nanfang Hospital, a large, tertiary-level teaching hospital with 2,200 beds in Guangzhou, China. 


\section{Bacterial Isolates}

To screen for carbapenem non-susceptible GNB, approximately $0.5 \mathrm{~mL}$ or $0.5 \mathrm{~g}$ of stool sample was suspended in $0.5 \mathrm{~mL}$ of $0.9 \%$ sterile saline, and $10 \mu \mathrm{L}$ of the resulting suspension was inoculated onto MacConkey agar medium (Beijing Land Bridge Technology, Beijing, China) containing 2 mg/L meropenem (Dainippon Sumitomo Pharma, Osaka, Japan) at $37^{\circ} \mathrm{C}$ for $18-24 \mathrm{~h}$. Colonies surviving on MacConkey agar medium were stored at $-80{ }^{\circ} \mathrm{C}$ in nutrient broth containing $30 \%$ (v/v) glycerol. The study was approved by the Medical Ethics Committee of Nanfang Hospital Southern Medical University and conducted in compliance with the Declaration of Helsinki.

\section{Species identification and anti-microbial susceptibility testing}

Species identification and anti-microbial susceptibility testing was conducted with the BD Phoenix 100 Automated Microbiology System (Becton Dickinson and Co., Franklin Lakes, NJ, USA), and the results were interpreted according to Clinical and Laboratory Standards Institute (CLSI) guidelines categories and minimum inhibitory concentration (MIC) breakpoints. Amplification and sequencing of a 996 base pair fragment from the partial 16S ribosomal RNA (rRNA) gene sequence were performed to confirm the genus or species level of the low confidence ( $<90$ value) value isolates. A standard nucleotide BLAST search of the NCBI sequence database (https://blast.ncbi.nlm.nih.gov/Blast.cgi) was performed to identify related $16 \mathrm{~S}$ rRNA sequences. E. coli ATCC 25922 and $P$. aeruginosa ATCC 27853 were used as control strains.

\section{Investigation of carbapenemase genes and the colistin resistance genes}

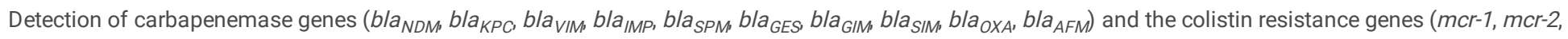
$m c r-3, m c r-4, m c r-5)$ were performed with polymerase chain reaction (PCR) followed by sequencing. Total DNA was amplified with the primers shown in Supplementary Table 1. Positive PCR amplifications were sequenced at the Beijing Genomics Institute (Shenzhen, China), and the sequences were compared with the NCBI database (www.ncbi.nlm.nih.gov).

\section{Conjugation Assay}

Conjugation experiments were performed in broth by using a sodium azide-resistant E.coli strain J53 as the recipient. The transconjugants were selected on Mueller-Hinton (MH) agar plates containing $4 \mu \mathrm{g} / \mathrm{ml}$ meropenem and $150 \mu \mathrm{g} / \mathrm{ml}$ sodium azide and MH agar plates supplemented with $4 \mu \mathrm{g} / \mathrm{ml}$ colistin and $150 \mu \mathrm{g} / \mathrm{ml}$ sodium azide. The MICs of imipenem, meropenem, ceftazidime, aztreonam and colistin against the donor, recipient and transconjugants were determined by using E-test strips (BioMérieux SA, La Balme-les-Grottes, France) obtained from Tian Kangxin (Beijing) Technology Co. Ltd. (Beijing, China) on $\mathrm{MH}$ agar plates.

\section{Whole genome sequencing and analysis}

It has been reported that the bla ${ }_{N D M}$ gene and $m c r$ gene were mainly existed in $E$. coli isolated from different sources, but the co-existence of $b / a_{N D M}$ gene and mor gene in K. pneumoniae was rarely reported. In our study, one K.pneumoniae strain carrying blaNDM- 5 gene and $m c r-1$ gene was detected in human feces, and which was resistant to carbapenem and colistin (Supplementary Table 2). Thus, the strain was selected for the whole genome sequencing. The whole genome was sequenced using the Single Molecule Real-Time (SMRT) sequencing platform with the PacBio sequencer and Illumina HiSeq at the Health Time Gene Institute (Shenzhen, China). The reads were denovo assembled using the HGAP (version 3.0). The prediction and annotation of the genome was achieved using GeneMarkS (version 4.6b, http://topaz.gatech.edu/GeneMark/) and BLAST (https://blast.ncbi.nlm.nih.gov/). The plasmids were typed using the PlasmidFinder 2.1 (https://cge.cbs.dtu.dk/services/PlasmidFinder/). The comparative and synteny analysis were generated using by BLAST and MUMmer (version 3.23, http://mummer.sourceforge.net/).

\section{Clinical data collection}

The electronic medical records system were reviewed systematically for the clinical characteristics of the patients carrying fecal CNS-GNB. The following information was included: demographics (age and sex); geographical distribution of patients; colonization/infection status, type of infection, therapy received and clinical outcome; Data on transplantation and immunosuppression, prior receipt of glucocorticoid, prior exposure to carbapenems, prior hospitalization, and invasive procedures such as surgery, use of mechanical ventilation, use of peripherally inserted central catheter were also recorded.

\section{Results}

\section{Identification of isolates}

A total of 745 samples harboring CNS-GNB were selected from 10,000 fecal samples, and 680 CNS-GNB were identified in 654 samples excluding 91 samples with S. maltophilia only (Supplementary Fig. 1). A total of 22 genera were identified, including nine types of 504 (74.1\%) carbapenem-resistant non-fermenting Bacillus, eight types of 156 (22.9\%) carbapenem-resistant Enterobacteria, and four types of 20 (2.9\%) other CR-GNB. The genera isolated in the highest proportion were Acinetobacter spp. (230/680, 33.8\%), Pseudomonas spp. (171/680, 25.1\%), and Enterobacter spp. (61/680, 9.0\%) (Table 1). 
Table 1

The carbapenemase genes and colistin-resistant genes of 680 carbapenem-non-susceptible GNE

\begin{tabular}{|c|c|c|c|c|c|c|c|c|c|}
\hline \multirow[t]{2}{*}{ Class } & \multirow[t]{2}{*}{ Genus } & \multirow[t]{2}{*}{ Species } & \multicolumn{7}{|c|}{ No. of isolates } \\
\hline & & & $\begin{array}{l}\text { Total } \\
(n= \\
680)\end{array}$ & $\begin{array}{l}\text { Carpenmase } \\
\text { genes } \\
(n=307)\end{array}$ & $\begin{array}{l}\text { NDM-1 } \\
(n= \\
188)\end{array}$ & $\begin{array}{l}\text { NDM-4 } \\
(n=2)\end{array}$ & $\begin{array}{l}\text { NDM-5 } \\
(n=15)\end{array}$ & $\begin{array}{l}\text { NDM-7 } \\
(n=1)\end{array}$ & $\begin{array}{l}\text { KPC- } \\
(n=7)\end{array}$ \\
\hline \multirow{25}{*}{$\begin{array}{l}\text { Carbapenem- } \\
\text { resistant non- } \\
\text { fermenting } \\
\text { bacillus } \\
(n=504)\end{array}$} & \multirow{6}{*}{$\begin{array}{l}\text { Acinetobacter } \\
(n=230)\end{array}$} & Acinetobacter baumannii & 42 & 9 & 9 & & & & \\
\hline & & Acinetobacter junii & 25 & 6 & 6 & & & & \\
\hline & & Acinetobacter johnsonii & 14 & 5 & 5 & & & & \\
\hline & & Acinetobacter calcoaceticus & 15 & 2 & 2 & & & & \\
\hline & & $\begin{array}{l}\text { Acinetobacter } \\
\text { Iwoffii/haemolyticus }\end{array}$ & 3 & & & & & & \\
\hline & & Acinetobacterspp.* & 131 & 115 & 115 & & & & \\
\hline & \multirow{2}{*}{$\begin{array}{l}\text { Alcaligenes } \\
(n=4)\end{array}$} & Alcaligenes faecalis & 3 & 1 & 1 & & & & \\
\hline & & Alcaligenes spp.* & 1 & & & & & & \\
\hline & $\begin{array}{l}\text { Achromobacter } \\
(\mathrm{n}=22)\end{array}$ & Achromobacterspp. & 22 & 10 & 1 & & & & \\
\hline & \multirow{2}{*}{$\begin{array}{l}\text { Burkholderia } \\
(\mathrm{n}=28)\end{array}$} & Burkholderia cepacia & 26 & 1 & 1 & & & & \\
\hline & & Burkholderia spp.* & 2 & & & & & & \\
\hline & \multirow{2}{*}{$\begin{array}{l}\text { Chryseobacterium } \\
(\mathrm{n}=4)\end{array}$} & Chryseobacterium indologenes & 1 & & & & & & \\
\hline & & Chryseobacterium spp.* & 3 & & & & & & \\
\hline & \multirow{3}{*}{$\begin{array}{l}\text { Comamonas } \\
(n=31)\end{array}$} & Comamonas testosteroni & 11 & 2 & & & & & \\
\hline & & Comamonas aquatica & 5 & 1 & & & & & \\
\hline & & Comamonas spp.* & 15 & 3 & & & & & \\
\hline & Delftia & Delftia acidovorans & 6 & & & & & & \\
\hline & $(n=6)$ & & & & & & & & \\
\hline & \multirow{5}{*}{$\begin{array}{l}\text { Pseudomonas } \\
(n=171)\end{array}$} & Pseudomonas aeruginosa & 45 & 16 & 1 & & & & 1 \\
\hline & & Pseudomonas alcaliphila & 1 & & & & & & \\
\hline & & Pseudomonas putida & 102 & 41 & 4 & & & & \\
\hline & & Pseudomonas pseudoalcaligenes & 2 & 2 & & & & & \\
\hline & & Pseudomonas spp.* & 21 & 6 & 1 & 1 & & & \\
\hline & \multirow{2}{*}{$\begin{array}{l}\text { Sphingomonas } \\
(\mathrm{n}=8)\end{array}$} & Sphingomonas paucimobilis & 7 & & & & & & \\
\hline & & Sphingobacterium spiritovorum & 1 & & & & & & \\
\hline \multirow{9}{*}{$\begin{array}{l}\text { Carbapenem- } \\
\text { resistant } \\
\text { enterobacteriaceae } \\
(n=156)\end{array}$} & \multirow{2}{*}{$\begin{array}{l}\text { Bordetella } \\
(\mathrm{n}=8)\end{array}$} & Bordetella spp.* & 7 & 1 & & & & & \\
\hline & & Bordetella avium & 1 & & & & & & \\
\hline & Cedeces & Cedeces spp. & 2 & & & & & & \\
\hline & $(n=2)$ & & & & & & & & \\
\hline & $\begin{array}{l}\text { Citrobacter } \\
(n=14)\end{array}$ & Citrobacter freundii & 14 & 9 & 9 & & & & \\
\hline & $\begin{array}{l}\text { Escherichia } \\
(\mathrm{n}=27)\end{array}$ & Escherichia coli & 27 & 19 & 4 & 1 & 13 & 1 & \\
\hline & \multirow{3}{*}{$\begin{array}{l}\text { Enterobacter } \\
(n=34)\end{array}$} & Enterobacter aerogenes & 12 & 5 & & & & & \\
\hline & & Enterobacter cloacae & 21 & 11 & 2 & & & & \\
\hline & & Enterobacter ludwigii & 1 & 1 & 1 & & & & \\
\hline
\end{tabular}

*species belong to the genus other than those listed 


\begin{tabular}{|c|c|c|c|c|c|c|c|}
\hline & $\begin{array}{l}\text { Klebsiella } \\
(\mathrm{n}=41)\end{array}$ & Klebsiella pneumoniae & 41 & 24 & 12 & 2 & 6 \\
\hline & $\begin{array}{l}\text { Proteus } \\
(\mathrm{n}=7)\end{array}$ & Proteus mirabilis & 7 & 2 & & & \\
\hline & Providencia & Providencia rettgeri & 17 & 11 & 11 & & \\
\hline & $(n=22)$ & Providencia vermicola & 5 & 2 & 2 & & \\
\hline & $\begin{array}{l}\text { Serratia } \\
(n=1)\end{array}$ & Serratia marcescens & 1 & & & & \\
\hline \multirow{9}{*}{$\begin{array}{l}\text { Other gram- } \\
\text { negative bacilli } \\
(n=20)\end{array}$} & \multirow{5}{*}{$\begin{array}{l}\text { Aeromonas } \\
(n=11)\end{array}$} & Aeromonas sobria & 6 & & & & \\
\hline & & Aeromonas hydrophila & 1 & & & & \\
\hline & & Aeromonas caviae & 2 & 1 & & & \\
\hline & & Aeromonas veronii & 1 & & & & \\
\hline & & Aeromonas spp.* & 1 & & & & \\
\hline & \multirow{2}{*}{$\begin{array}{l}\text { Cupriavidus } \\
(n=2)\end{array}$} & Cupriavidus gilardii & 1 & 1 & 1 & & \\
\hline & & Cupriavidus pauculus & 1 & 1 & & & \\
\hline & $\begin{array}{l}\text { Empedobacter } \\
(\mathrm{n}=5)\end{array}$ & Empedobacter brevis & 5 & & & & \\
\hline & $\begin{array}{l}\text { Pasteurella } \\
(\mathrm{n}=2)\end{array}$ & Pasteurella pneumotropica & 2 & & & & \\
\hline
\end{tabular}

\section{Antimicrobial susceptibility testing}

Most of the isolates were resistant to cephalosporin, but they were sensitive to colistin. Amikacin resistance was identified in 147 isolates (22.5\%). Most of the carbapenem-resistant non-fermenting Bacillus had a sensitivity rate of more than $80 \%$, and sensitivity to amikacin of $A$. baumannii was $54.8 \%$. Most of CRE strains had a resistance rate of less than $10 \%$ to amikacin; E.coli had a resistance rate of $25.8 \%$. A total of $490(75.2 \%)$ isolates were ciprofloxacin resistant, 391 (57.5\%) isolates were levofloxacin resistant, and the susceptibility of different CRE species to ciprofloxacin and levofloxacin varied. CRE species were more sensitive to trimethoprim/sulfamethoxazole than non-fermenting Bacillus, and 527 (77.5\%) isolates showed resistance to trimethoprim/sulfamethoxazole. A total of 354 (54.3\%) isolates were resistant to tetracycline, and non-fermenting Bacillus species were more sensitive to tetracycline than CRE (Table 2). 
Table 2

a Susceptibility (SIR) pattern of carbapenem-resistant non-ferment

\begin{tabular}{|c|c|c|c|c|c|c|c|c|c|c|c|c|c|}
\hline & \multicolumn{13}{|c|}{ Susceptibility SIR (\%) } \\
\hline & \multicolumn{3}{|c|}{$\begin{array}{l}\text { Acinetobacter spp. } \\
(n=131)\end{array}$} & \multicolumn{3}{|c|}{$\begin{array}{l}\text { Acinetobacter junii } \\
(\mathrm{n}=25)\end{array}$} & \multicolumn{3}{|c|}{$\begin{array}{l}\text { Acinetobacter } \\
\text { baumannii } \\
(n=42)\end{array}$} & \multicolumn{3}{|c|}{$\begin{array}{l}\text { Pseudomonas } \\
\text { putida } \\
(n=102)\end{array}$} & \multirow{2}{*}{$\begin{array}{l}P s \\
a e \\
\text { (n } \\
\text { S }\end{array}$} \\
\hline & $\mathrm{S}$ & I & $\mathbf{R}$ & S & I & $\mathbf{R}$ & S & I & $\mathbf{R}$ & S & I & $\mathbf{R}$ & \\
\hline Amikacin & 84.0 & 9.2 & 6.9 & 80.0 & 0.0 & 20.0 & 54.8 & 12.9 & 32.3 & 92.2 & 3.9 & 3.9 & 82. \\
\hline Gentamicin & 19.8 & 6.9 & 73.3 & 20.0 & 0.0 & 80.0 & 45.2 & 0.0 & 54.8 & 21.6 & 1.0 & 76.5 & 73. \\
\hline Imipenem & 0.0 & 0.0 & 100.0 & 0.0 & 0.0 & 100.0 & 0.0 & 0.0 & 100.0 & 2.0 & 5.9 & 92.2 & 0.0 \\
\hline Meropenem & 0.0 & 0.0 & 100.0 & 0.0 & 0.0 & 100.0 & 0.0 & 0.0 & 100.0 & 5.9 & 3.9 & 90.2 & 4.4 \\
\hline Colistin & 96.2 & - & 3.8 & 100.0 & - & 0.0 & 100.0 & - & 0.0 & - & - & - & 10( \\
\hline Trimethoprim/sulfamethoxazole & 23.7 & - & 75.6 & 48.0 & - & 52.0 & 61.3 & - & 38.7 & 15.7 & - & 84.3 & - \\
\hline Chloramphenicol & - & - & - & - & - & - & - & - & - & 0.0 & 0.0 & 100.0 & - \\
\hline Ciprofloxacin & 9.9 & 2.3 & 87.8 & 84.0 & 0.0 & 16.0 & 38.7 & 0.0 & 61.3 & 9.8 & 0.0 & 90.2 & 28. \\
\hline Levofloxacin & 47.3 & 29.8 & 22.9 & 68.0 & 16.0 & 16.0 & 38.7 & 61.3 & 0.0 & 9.8 & 5.9 & 84.3 & 73. \\
\hline Tetracycline & 34.4 & 38.2 & 28.2 & 68.0 & 16.0 & 16.0 & 80.6 & 19.4 & 0.0 & 20.6 & 11.8 & 66.7 & - \\
\hline
\end{tabular}

\section{Table 2}

b Susceptibility (SIR) pattern of carbapenem-resistant enterobacteriaceae

\begin{tabular}{|c|c|c|c|c|c|c|c|c|c|}
\hline & \multicolumn{9}{|c|}{ Susceptibility SIR (\%) } \\
\hline & \multicolumn{3}{|c|}{$\begin{array}{l}\text { Escherichia coli } \\
(\mathrm{n}=27)\end{array}$} & \multicolumn{3}{|c|}{$\begin{array}{l}\text { Enterobacter cloacae } \\
(\mathrm{n}=21)\end{array}$} & \multicolumn{3}{|c|}{$\begin{array}{l}\text { Klebsiella pneumoniae } \\
(\mathrm{n}=41)\end{array}$} \\
\hline & $S$ & 1 & $\mathrm{R}$ & S & 1 & $\mathrm{R}$ & $\mathrm{S}$ & 1 & $\mathrm{R}$ \\
\hline Amikacin & 74.1 & 0.0 & 25.9 & 85.7 & 4.8 & 9.5 & 95.1 & 0.0 & 4.9 \\
\hline Gentamicin & 37.0 & 0.0 & 63.0 & 66.7 & 0.0 & 33.3 & 80.5 & 0.0 & 19.5 \\
\hline Imipenem & 0.0 & 29.6 & 70.4 & 0.0 & 9.5 & 90.5 & 0.0 & 9.8 & 90.2 \\
\hline Meropenem & 0.0 & 11.1 & 88.9 & 0.0 & 14.3 & 85.7 & 0.0 & 9.8 & 90.2 \\
\hline Colistin & 100.0 & - & 0.0 & 100.0 & - & 0.0 & 97.6 & - & 2.4 \\
\hline Trimethoprim/sulfamethoxazole & 44.4 & - & 55.6 & 76.2 & - & 23.8 & 73.2 & - & 26.8 \\
\hline Chloramphenicol & 33.3 & 3.7 & 63.0 & 0.0 & 0.0 & 100.0 & 0.0 & 0.0 & 100.0 \\
\hline Ciprofloxacin & 44.4 & 3.7 & 51.9 & 76.2 & 0.0 & 23.8 & 73.2 & 2.4 & 24.4 \\
\hline Levofloxacin & 14.8 & 14.8 & 70.4 & 81.0 & 9.5 & 9.5 & 46.3 & 2.4 & 51.2 \\
\hline Tetracycline & 3.7 & 0.0 & 96.3 & 57.1 & 0.0 & 42.9 & 9.8 & 4.9 & 85.4 \\
\hline \multicolumn{10}{|c|}{ Drug susceptibility according to Clinical and Laboratory Standards Institute (CLSI) M100-S29 criteria. '-', not applicable. } \\
\hline
\end{tabular}

\section{Investigation of carbapenemase and colistin resistance genes}

A total of 307 (45.1\%) isolates harbored carbapenemase genes. Among them, bla $a_{\text {NDM }}$ was detected in $206(67.1 \%)$ isolates, including $188(61.2 \%)$ bla ${ }_{N D M-1}, 2$ $(0.7 \%) b / a_{N D M-4}, 15$ (4.9\%) bla $a_{N D M-5}$, and one (0.3\%) bla $a_{N D M-7}$. In addition, 51 (16.6\%) isolates harbored the bla ${ }_{V I M-2}$ gene, and $b / a_{I M P}$ and $b / a_{K P C-2}$ were observed in 48 (15.6\%) and seven (2.3\%) isolates, respectively. Four isolates were found to co-exist with the $b / a_{I M P}$ and $b / a_{K P C}$ genes. Seven isolates were found to harbor the bla AFM-1 $_{1}$ gene, a novel metallo- $\beta$-lactamase gene; these isolates include Alcaligenes faecalis, Bordetella trematum, Comamonas testosterone, and Comamonas aquatica. Five strains of Acinetobacter and one strain of $K$. pneumoniae were positive for the colistin-resistant gene mcr- 1 and 
co-existed with the bla $a_{\mathrm{NDM}}$ gene. No other carbapenemase-related and colistin resistance genes were identified (Table 1). Acinetobacter spp. was the most abundant carbapenemase-producing species (115/131, 87.8\%), followed by E. coli (19/27, 70.4\%), P. rettgeri (11/17, 64.7\%), C. freundii (9/14, 64.3\%), and K. pneumoniae $(24 / 41,58.5 \%)$. P. aeruginosa and $K$. pneumoniae had the most carbapenemase gene types; $b_{N D M-1}$ was the most common carbapenemase gene in Acinetobacterspp. $(115 / 115,100.0 \%), b_{N D M-5}$ was the most common type in $E$. coli $(13 / 19,68.4 \%)$, and $b / a_{K P C-2}$ was the most common in $K$.

\section{pneumoniae.}

\section{Clinical data collection}

Of 654 unique individuals with CR-GNB, 549 (83.9\%) were from Southern China, mainly Guangdong province (534/549, 97.3\%). The highest proportion was from Guangzhou in Guangdong (Supplementary Fig. 2); 71 (10.9\%) were from Central China, 16 (2.4\%) from Eastern China; and 18 (2.8\%) from other regions in China. A total of 415 (63.5\%) were men and 239 (36.5\%) were women and the median age was 55 years (range, < 1-91 years; Table 3 ). A total of 653 $(99.8 \%)$ had at least one underlying comorbid condition with a median Charlson comorbidity index of 2 (range, $0-4)$ and 1 ( $0.2 \%$ ) had no documented underlying condition. The most commonly reported conditions included diabetes $(99,15.1 \%)$ and neurological disorders (188, $28.7 \%)$; the ratio of malignancy $(104,15.9 \%)$ and chronic pulmonary disease $(88,13.5 \%)$ also surpassed other general underlying diseases. Bacterial infections were prominent, and most of the cases had a history of antibiotic use. 
Table 3

Demographic and underlying condition of Individuals with carbapenem nonsusceptible Gram-negative bacilli

\begin{tabular}{|c|c|}
\hline & No./Total (\%) \\
\hline \multicolumn{2}{|l|}{ Demographic } \\
\hline \multicolumn{2}{|l|}{ Age (years) } \\
\hline Male & $415 / 654(63.5)$ \\
\hline Female & $239 / 654(36.5)$ \\
\hline \multicolumn{2}{|l|}{ Age group, y } \\
\hline $0-18$ & $44(6.7)$ \\
\hline $19-44$ & $157(24.0)$ \\
\hline $45-59$ & 199(30.4) \\
\hline $60-79$ & $217(33.2)$ \\
\hline$\geqq 80$ & $37(5.7)$ \\
\hline Age, median (range) & $55(<1-91)$ \\
\hline \multicolumn{2}{|l|}{ Underlying condition } \\
\hline None & $1 / 654(0.2)$ \\
\hline Chronic pulmonary disease & $88 / 654(13.5)$ \\
\hline Chronic renal insufficiency & 29/654(4.4) \\
\hline Bacterial infections ${ }^{a}$ & $204 / 654(31.2)$ \\
\hline Neurological disorder & $188 / 654(28.7)$ \\
\hline Hepatobiliary diseases & $34 / 654(5.2)$ \\
\hline Cirrhosis & $1 / 654(0.2)$ \\
\hline Viral hepatitis & $60 / 654(9.2)$ \\
\hline Myocardial infarction & $12 / 654(1.8)$ \\
\hline Connective tissue disease & $9 / 654(1.4)$ \\
\hline Hematological diseases & $57 / 654(8.7)$ \\
\hline Diabetes & 99/654(15.1) \\
\hline Any malignancy & 104/654(15.9) \\
\hline Transplant recipient & $2 / 654(0.3)$ \\
\hline Immunodeficiency related diseases & $1 / 654(0.2)$ \\
\hline Other & $99 / 654(15.1)$ \\
\hline Charlson Comorbidity Index, median(range) ${ }^{\mathrm{b}}$ & $2(0-4)$ \\
\hline \multicolumn{2}{|l|}{ Previous exposure to healthcare } \\
\hline History of antibiotic use & $144 / 654(22.0)$ \\
\hline Immunosuppressive medications in past 30 days & $33 / 654(5.0)$ \\
\hline Hospitalized within one year(except this time) & $296 / 654(45.3)$ \\
\hline Radiotherapy or chemotherapy many times & $62 / 654(9.5)$ \\
\hline Surgery within one year & $54 / 654(8.3)$ \\
\hline Current maintenance dialysis & $5 / 654(0.8)$ \\
\hline Trachoestomy & $2 / 654(0.3)$ \\
\hline \multicolumn{2}{|l|}{${ }^{\mathrm{a}}$ In addition to gastrointestinal infectious diseases } \\
\hline \multicolumn{2}{|c|}{ bScore range is 0 to 37 ; the higher the number, the more serious the constellatio } \\
\hline of coexisting comorbidities. & \\
\hline
\end{tabular}

Page $8 / 15$ 


\section{Conjugation}

The transconjugants were selected on screening plates. PCR and sequencing by Sangon Biotech Co. Ltd. (Shanghai, China) revealed that the transconjugant NFYY0065-N contained the $b / a_{N D M-5}$ gene, and the transconjugant NFYY0065-M contained the $m c r-1$ gene. Antimicrobial susceptibility testing showed that NFYY0065-N was resistant to imipenem, meropenem, ceftazidime, and aztreonam, whereas NFYY0065-M was resistant to colistin (Table 4).

Table 4

MICs of imipenem, meropenem, ceftazidime, aztreonam and colistin against the donor, recipient and transconjugants.

\begin{tabular}{|lllllll|}
\hline Strain(s) & Description & \multicolumn{3}{l}{ MIC $(\mu \mathrm{g} / \mathrm{ml})$} & & \\
\cline { 4 - 7 } & & imipenem & meropenem & ceftazidime & aztreonam & colistin \\
\hline NFYY0065 & donor & 32 & 32 & 256 & 48 & 8 \\
\hline E.coli strain J53 & recipient & 0.25 & 0.064 & 0.125 & 0.064 & 0.25 \\
\hline NFYY0065-N & Transconjugant containing bla NDM-5 $_{n}$ & 8 & 8 & 128 & 32 & 0.5 \\
\hline NFYY0065-M & Transconjugant containing mcr-1 & 0.25 & 0.064 & 0.125 & 0.064 & 4 \\
\hline
\end{tabular}

\section{Genomic analysis of CRKP co-producing bla $\mathrm{NDM}_{-5}$ and mcr-1}

The chromosome was 5,235,159 bp with $57.55 \%$ GC content; it harbored 5186 genes, including five drug resistance genes encoding a class A broad-spectrum beta-lactamase, multidrug efflux transporter transcriptional repressor, tetracycline repressor protein, multidrug efflux MFS transporter, and fosfomycin resistance protein (Table 5).

Table 5

The antibiotic-resistance genes located on chromosome and plasmids in Klebsiella pneumoniae strain NFYY0065.

\begin{tabular}{|c|c|c|c|}
\hline Sequence(s) & Size (bp) & $G+C(\%)$ & Resistance gene(s) \\
\hline Chromosome & $5,235,159$ & 57.55 & bla $_{S H V-1}, a c r R$, tetR, emrD, fosA \\
\hline Plasmid pAN65-3 & 46,161 & 46.65 & bla $_{N D M-5}$, bleMBL \\
\hline Plasmid pAN65-1 & 276,370 & 46.31 & 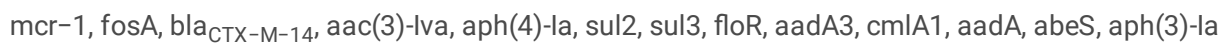 \\
\hline
\end{tabular}

pAN65-MCR was 276,370 bp, possessed a typical IncHI2-type backbone, and contained 319 predicted open reading frames. It harbored the $m c r-1$ gene, which was located between the IS30-like element ISApl1 and a PAP2-like encoding gene (Fig. 1). The plasmid co-carried the resistance gene blaCTX-M-14. BLASTn analysis of the complete nucleotide sequence against the NCBI database indicated the highest similarity ( $95 \%$ query cover and $99 \%$ nucleotide identity) to plasmid pMCR_WCHEC050613 (CP019214.2), which was from E. coli isolated from sewage in Chengdou. The genetic structure of mcr-1 in plasmid PMCR_WCHEC050613 was also the same as pAN65-MCR. BLAST homology analyses revealed that pAN65-MCR has $93 \%$ identity and $99 \%$ query coverage with pHNSHP45 [8], which was isolated from pigs. Comparative analysis of the genetic structures of $m c r-1$ in the reference plasmids pHN6DS2 and pMCR1-PA revealed there were identical to pAN65-1, and pHNSHP45 had lost the PAP2 gene downstream of $m c r$ (Fig. 2).

The bla NDM- -bearing plasmid pAN65-NDM was 46,161 bp with a GC content of $46.65 \%$ and belonged to the IncX3 group (Fig. 3 ). BLASTn analysis revealed high similarity $\left(100 \%\right.$ query cover and $99 \%$ nucleotide identity) to previously reported $b / a_{N D M-5}$-positive plasmids, including the $b / a_{N D M-5}$ positive plasmids of various sources, such as MH234502 (human, China) [9], CP027204 (sewage, China) [9], CP029245 (laying hen, China) [9], CP028705 (vegetable, China) [9], KX507346 (swine, China) [9], and CP034744 (goose, China) [9]. Comparative analysis revealed that these plasmids are highly similar, possessing the same backbone, which includes IncX3 replication. The genetic structure of the $b / a_{N D M-5}$ gene was identical to the plasmids pNDM5_020038, pK516_NDM5, and tig00001251; the mobile genetic elements IS30 and IS5 were upstream of bla ${ }_{N D M-5}$ and the genes bleMBL and trpF were downstream (Fig. 4).

\section{Discussion}

Intestinal flora forms a major reservoir of bacteria, including multidrug-resistant bacteria. In this study, the overall prevalence of fecal carriage of CNS-GNB in 10,000 stool samples was $7.45 \%$, and the rate of carrying CRE was $1.6 \%$, of which the dominant species were $K$. pneumoniae and E. coli, consistent with a previous report [10]. This showed that K. pneumoniae and E. coli were the dominant species in $60 \mathrm{CRE}$ isolated from 704 stool samples. However, the rate of feces carrying CRE was $8.5 \%$; this may be due to the fact that the stool samples collected in this study were primarily from hospitalized patients in Intensive Care Unit. The fecal samples collected in the present study contained 5.0\% carbapenem-resistant non-fermenting bacillus, and Acinetobacter spp. was the dominant species accounting for $2.3 \%$; this result was similar to our previous report of the positive rate of Acinetobacter spp. [11]. In general, the abundance of Enterobacteriaceae in the normal intestine is greater than Acinetobacter, but the positive rate of carbapenem-resistant Acinetobacter in the intestine was higher than Enterobacteriaceae in our study. Acinetobacter is the main pathogenic bacteria that causes nosocomial infections and is widely distributed in the 
environment, which is significant for the spread of drug-resistant bacteria. We isolated 22 genera of CR-GNB from stool samples, some of which did not cause clinical infections, suggesting the possibility that drug resistance had spread widely between different strains.

CR-GNB isolates in the present study exhibited high resistance rates to $\beta$-lactam antimicrobial agents, including cephalosporins, carbapenems, and the monobactam aztreonam; however, most isolates were sensitive to colistin. The susceptibility of different CRE species to amikacin varied; most of the isolates exhibited low resistance to amikacin (<20.0\%). Liu et al. [10] reported that resistance to amikacin against CRE species isolated from fecal samples was less than $25 \%$, while Wang et al. [12] reported that 1801 clinical CRE isolates showed susceptibility to amikacin (54.5\%). Carbapenem-resistant strains isolated from fecal survey samples were more sensitive to amikacin than clinical strains. Pritsch et al. [13] reported that two of three carbapenamase-producing $A$. baumannii isolates were untreatable with locally available antimicrobial agents and were susceptible to colistin and amikacin only. In some cases, Our datas provided an effective choice for clinical treatment of infections.

A total of 307 (45.1\%) isolates carried carbapenemase genes. bla $a_{N D M}(67.1 \%, 206 / 307)$ was the predominant carbapenemase gene in the present study; the four main types included $b / a_{N D M-1}, b / a_{N D M-4}, b / a_{N D M-5}$, and $b / a_{N D M-7}$. The positive rate of the bla $a_{N D M-1}$ gene was higher than for any other variant, consistent with previous reports [14]. In the present study, $b / a_{N D M}$ was identified in different species, and the sequencing of four different species indicated that $b / a_{N D M}$ can be transmitted between different species through mobile genetic elements or removable plasmids, including Enterobacteriaceae and non-fermenting Bacillus. Notably, NDM was mainly present in Acinetobacter, and we detected only one type of carbapenemase NDM in Acinetobacter, indicating that NDM production is an important factor in the resistance of Acinetobacter to carbapenem. Other metal enzymes such as VIM and IMP were mainly present in Pseudomonas spp., and KPC was mainly present in K. pneumoniae. Apart from the NDM, KPC, VIM, and IMP carbapenemases, no other carbapenemase genes were found. OXA-48, a carbapenemase gene found in many European countries, is rare in China. Whether the results of the present study, and those of previous studies in which OXA-48 was not detected, are typical, will require further investigation [15, 16]. In addition, 373 carbapenemase gene-negative GNB isolates were identified. Other mechanisms of carbapenem resistance have been recognized, which include overexpression of Extended Spectrum BetaLactamases (ESBLs) or AmpC $\beta$-lactamase, decreased membrane permeability due to porin loss, and expression of efflux pumps [17, 18]. The complexity of carbapenem resistance mechanisms in GNB and characteristics of the human gastrointestinal tract make the dissemination and transmutation of resistance genes more complex and frequent.

The $b / a_{A F M}$ gene was discovered in our research. This gene is a novel metallo- $\beta$-lactamase gene; its expression product can hydrolyze carbapenemase. Therefore, strains carrying the $b_{A F M}$ gene can also develop resistance to carbapenem antibiotics. In the present study, the $b_{A F M}$ gene was also present in different strains of the human gastrointestinal tract. Although it was not detected in common isolates of Enterobacteriaceae, the $b / a_{A F M}$ gene is often located on a plasmid and can spread through mobile genetic elements.

Colistin is a last-resort antimicrobial for infections caused by multidrug resistant Gram-negative bacteria. In 680 CNS-GNB, six isolates were resistant to colistin, which indicated low prevalence of the $\mathrm{mcr}-1$ gene among carbapenemase-producing fecal survey isolates. The $m c r-1$ gene is a transferable resistance determinant against colistin. We detected five strains of Acinetobacter and one strains of $K$. pneumoniae co-producing mcr- 1 and NDM- 5 in the present study. It has been reported that both $b / a_{N D M}$ and $m c r$ genes are commonly present in $E$. coli isolated from different sources, but the co-existence of the $b / a_{N D M}$ and morgenes in K. pneumoniae has rarely been reported. In this study, the plasmid harboring the morgene in $K$. pneumoniae was similar to other IncHI2-type plasmids present in E. coli, and upstream of the morgene was the common ISApl1 transposon. NDM-5 has been reported in many other countries, including India [19], China [20], Denmark [21], Italy [22], America [23], and Spain [24]; it has also been isolated from pigs [25, 26], dairy cows [27], and vegetables [28]. In the present study, the IncX3 plasmids harboring $b / a_{N D M-5}$ were highly similar in various sources, suggesting their ability to be an efficient vehicle for $b / a_{N D M-5}$ dissemination among humans, animals, food, and the environment via the human intestine. This strain can capture the plasmid harboring $m c r$ or $b / a_{N D M}$ genes, which leads to the generation of pan-drug resistance, and the phenomenon of co-existence of $m c r$ or $b^{\prime} a_{N D M}$ genes has spread to other isolates, which poses a significant challenge in the treatment of clinical infections.

In the present study, individuals with CRE were primarily from the pneumology department, hematology department, intensive care unit, and rehabilitation department. There were some common feature of individuals with CRE, most of them had infectious diseases, multiple hospital admissions and higher infectious risk, the history of using antibiotics. Therefore, the frequent use of antibiotics was closely related to multidrug resistant bacteria. However, some individuals with CRE did not have underlying diseases, and there was no record of previous antibiotic use. In addition, these individuals were from different regions of China, indicating that CRE has been widely present in the intestines of normal individuals. If individuals carrying resistance genes have infections, failure of antibiotic treatment, as well as an increase in the risk of transmission to other patients, is likely.

In conclusion, the emergence of CNS-GNB in fecal matter poses a major concern. Our data showed that the overall prevalence of fecal carriage of CNS-GNB in 10,000 stool samples was $7.45 \%$, Acinetobacter was the dominant isolate of CNS-GNB, and most of the strains were sensitive to colistin. The presence of carbapenemase genes was the main mechanism of CNS-GNB resistance to carbapenem antibiotics, mainly encoding NDM. Notably, bla ${ }_{N D M}$ genes were widely distributed in various isolates. The bla ${ }_{N D M}$ genes were first detected in Providencia vermicola, Achromobacter spp., and Cupriavidus gilardii, and Achromobacter co-producing $b / a_{V I M}$ and $b / a_{I M P}$ genes has not been reported. Genomic sequencing and analysis of $K$. pneumoniae harboring bla ${ }_{N D M}$ and $m c r$ revealed that these genes were located on two different plasmids, which were similar to previously reported plasmids, suggesting their ability to be efficient vehicles for $b / a_{N D M-5}$ dissemination among humans, animals, food, and the environment via the human intestine. Our results highlight the fact that enhanced surveillance and health policies for the detection and control of these pathogens are urgently needed to limit the emergence and spread of such an organism. As the final antibiotic for the treatment of CR-GNB infection, the emergence of MCR-carrying strains reminds us to strengthen the management of antibiotic use and encourages the implementation of human feces surveillance, as well as actions to prevent and control the spread of multidrug resistant bacteria, especially bacteria resistant to carbapenems and colistin. 


\section{Declarations}

Ethics approval and consent to participate: Verbal informed consent was obtained from all participants. The samples used were the specimens remaining after the patient's clinical examination. The study was approved by the Medical Ethics Committee of Nanfang Hospital Southern Medical University and conducted in compliance with the Declaration of Helsinki (No. NFEC-2014-002).

Consent to publish: Not applicable.

Availability of data and materials: All data generated or analyzed in this study are included in this published article and its supplementary information files.

Competing interests: The authors declare that they have no competing interests. Funding: This study was supported by grants from Guangdong Province Science and Technology Project (Nos. 2014A010107011 and 2015A020211011) and Guangzhou City Science and Technology (No. 201510010167).

Authors' contributions: Si Li carried out the experiments, data organization and analysis, and contributed to writing the manuscript and annotating the results. Xiaonv Duan collected all the isolates and clinical data. Yuan Peng participated in a subset of the experiments. Yongyu Rui contributed to the design of the study and assisted in drafting of the manuscript. All authors have read and approved the manuscript.

\section{References}

1. Codjoe FS, et al. Phenotypic and Genotypic Characterization of Carbapenem-Resistant Gram-Negative Bacilli Pathogens from Hospitals in Ghana. Microbial Drug Resistance. 2019;25(10):1449-57.

2. Yunus F, Yunus F, Izhar M. New Delhi Metallo-Beta-Lactamase Producing Carbapenem-Resistant Gram-Negative Bacilli: Microbiological And Genotypic Analyses At A Tertiary Care Hospital In Pakistan. J Ayub Med Coll Abbottabad. 2019;31(3):299-307.

3. Schill F, et al. Prevalence and characterization of extended-spectrum $\beta$-lactamase (ESBL) and AmpC $\beta$-lactamase producing Enterobacteriaceae in fresh pork meat at processing level in Germany. Int J Food Microbiol. 2017;257:58-66.

4. Yong $\mathrm{D}$, et al. Characterization of a new metallo-beta-lactamase gene, bla(NDM-1), and a novel erythromycin esterase gene carried on a unique genetic structure in Klebsiella pneumoniae sequence type 14 from India. Antimicrob Agents Chemother. 2009;53(12):5046-54.

5. Teo JW, et al. Emergence of a New Delhi metallo-beta-lactamase-1-producing Pseudomonas aeruginosa in Singapore. Emerg Microbes Infect. 2015;4(11):e72.

6. Liu YY, et al. Emergence of plasmid-mediated colistin resistance mechanism MCR-1 in animals and human beings in China: a microbiological and molecular biological study. Lancet Infect Dis. 2016;16(2):161-8.

7. Rebelo AR, et al., Multiplex PCR for detection of plasmid-mediated colistin resistance determinants, mcr-1, mcr-2, mcr-3, mcr-4 and mcr-5 for surveillance purposes. Euro Surveill, 2018. 23(6).

8. Liu YY, Wang Y, Walsh TR, et al. Emergence of plasmid-mediated colistin resistance mechanism MCR-1 in animals and human beings in China: a microbiological and molecular biological study. Lancet Infect Dis. 2016;16(2):161-8. doi:10.1016/S1473-3099(15)00424-7.

9. Tang B, Chang J, Cao L, et al. Characterization of an NDM-5 carbapenemase-producing Escherichia coli ST156 isolate from a poultry farm in Zhejiang, China. Bmc Microbiol. 2019;19(1):82. doi:10.1186/s12866-019-1454-2.

10. Liu Q, et al. Fecal Carriage and Epidemiology of Carbapenem-Resistant Enterobacteriaceae Among Hospitalized Patients in a University Hospital. Infection drug resistance. 2019;12:3935-42.

11. Li S, et al. Molecular characteristics of carbapenem-resistant Acinetobacter spp. from clinical infection samples and fecal survey samples in Southern China. BMC Infect Dis. 2019;19(1):900.

12. Wang Q, et al. Phenotypic and Genotypic Characterization of Carbapenem-resistantEnterobacteriaceae: Data From a Longitudinal Large-scale CRE Study in China (2012-2016). Clin Infect Dis. 2018;67(suppl_2):S196-205.

13. Pritsch $\mathrm{M}$, et al. First report on bla NDM-1-producing Acinetobacter baumannii in three clinical isolates from Ethiopia. BMC Infect Dis. 2017;17(1):180.

14. Boutal H, et al. Development and Validation of a Lateral Flow Immunoassay for Rapid Detection of NDM-Producing Enterobacteriaceae. J Clin Microbiol. 2017;55(7):2018-29.

15. Mairi A, et al. OXA-48-like carbapenemases producing Enterobacteriaceae in different niches. Eur J Clin Microbiol Infect Dis. 2018;37(4):587-604.

16. Nordmann P, Poirel L. The difficult-to-control spread of carbapenemase producers among Enterobacteriaceae worldwide. Clin Microbiol Infect. 2014;20(9):821-30.

17. Cacci LC, et al. Mechanisms of carbapenem resistance in endemic Pseudomonas aeruginosa isolates after an SPM-1 metallo-beta-lactamase producing strain subsided in an intensive care unit of a teaching hospital in Brazil. Mem Inst Oswaldo Cruz. 2016;111(9):551-8.

18. Tamma PD, et al. Comparing the Outcomes of Patients With Carbapenemase-Producing and Non-Carbapenemase-Producing Carbapenem-Resistant Enterobacteriaceae Bacteremia. Clin Infect Dis. 2017;64(3):257-64.

19. Rahman M, Shukla SK, Prasad KN, et al. Prevalence and molecular characterisation of New Delhi metallo- $\beta$-lactamases NDM-1, NDM-5, NDM-6 and NDM7 in multidrug-resistant Enterobacteriaceae from India. Int J Antimicrob Agents. 2014;44(1):30 - 7. doi:10.1016/j.ijantimicag.2014.03.003.

20. Zhang LP, Xue WC, Meng DY. First report of New Delhi metallo- $\beta$-lactamase 5 (NDM-5)-producing Escherichia coli from blood cultures of three leukemia patients. Int J Infect Dis. 2016;42:45-6. doi:10.1016/j.jid.2015.10.006.

21. Zhang LP, Xue WC, Meng DY. First report of New Delhi metallo- $\beta$-lactamase 5 (NDM-5)-producing Escherichia coli from blood cultures of three leukemia patients. Int J Infect Dis. 2016;42:45-6. doi:10.1016/j.ijid.2015.10.006.

Page $11 / 15$ 
22. Bitar I, Piazza A, Gaiarsa S, et al. ST405 NDM-5 producing Escherichia coli in Northern Italy: the first two clinical cases. Clin Microbiol Infect. 2017;23(7):489-90. doi:10.1016/j.cmi.2017.01.020.

23. Rojas LJ, Hujer AM, Rudin SD, et al. NDM-5 and OXA-181 Beta-Lactamases, a Significant Threat Continues To Spread in the Americas. Antimicrob Agents Chemother. 2017;61(7).doi:10.1128/AAC.00454-17.

24. Pérez-Moreno MO, Ortega A, Pérez-Vázquez M, et al. Simultaneous colonisation by ST340 Klebsiella pneumoniae producing NDM-5 and ST399 Escherichia coli producing NDM-7. Int J Antimicrob Agents. 2016;48(4):464-6. doi:10.1016/j.ijantimicag.2016.07.003.

25. Ho PL, Wang Y, Liu MC, et al. IncX3 Epidemic Plasmid Carrying bla(NDM-5) in Escherichia coli from Swine in Multiple Geographic Areas in China. Antimicrob Agents Chemother. 2018;62(3).doi:10.1128/AAC.02295-17.

26. Kong LH, Lei CW, Ma SZ, et al. Various Sequence Types of Escherichia coli Isolates Coharboring bla(NDM-5) and mcr-1 Genes from a Commercial Swine Farm in China. Antimicrob Agents Chemother. 2017;61(3).doi:10.1128/AAC.02167-16.

27. He T, Wang Y, Sun L, Pang M, Zhang L, Wang R. Occurrence and characterization of blaNDM-5-positive Klebsiella pneumoniae isolates from dairy cows in Jiangsu, China. J Antimicrob Chemother. 2017;72(1):90-4. doi:10.1093/jac/dkw357.

28. Liu BT, Zhang XY, Wan SW, Hao JJ, Jiang RD, Song FJ. Characteristics of Carbapenem-Resistant Enterobacteriaceae in Ready-to-Eat Vegetables in China. Front Microbiol. 2018;9:1147. doi:10.3389/fmicb.2018.01147.

\section{Figures}

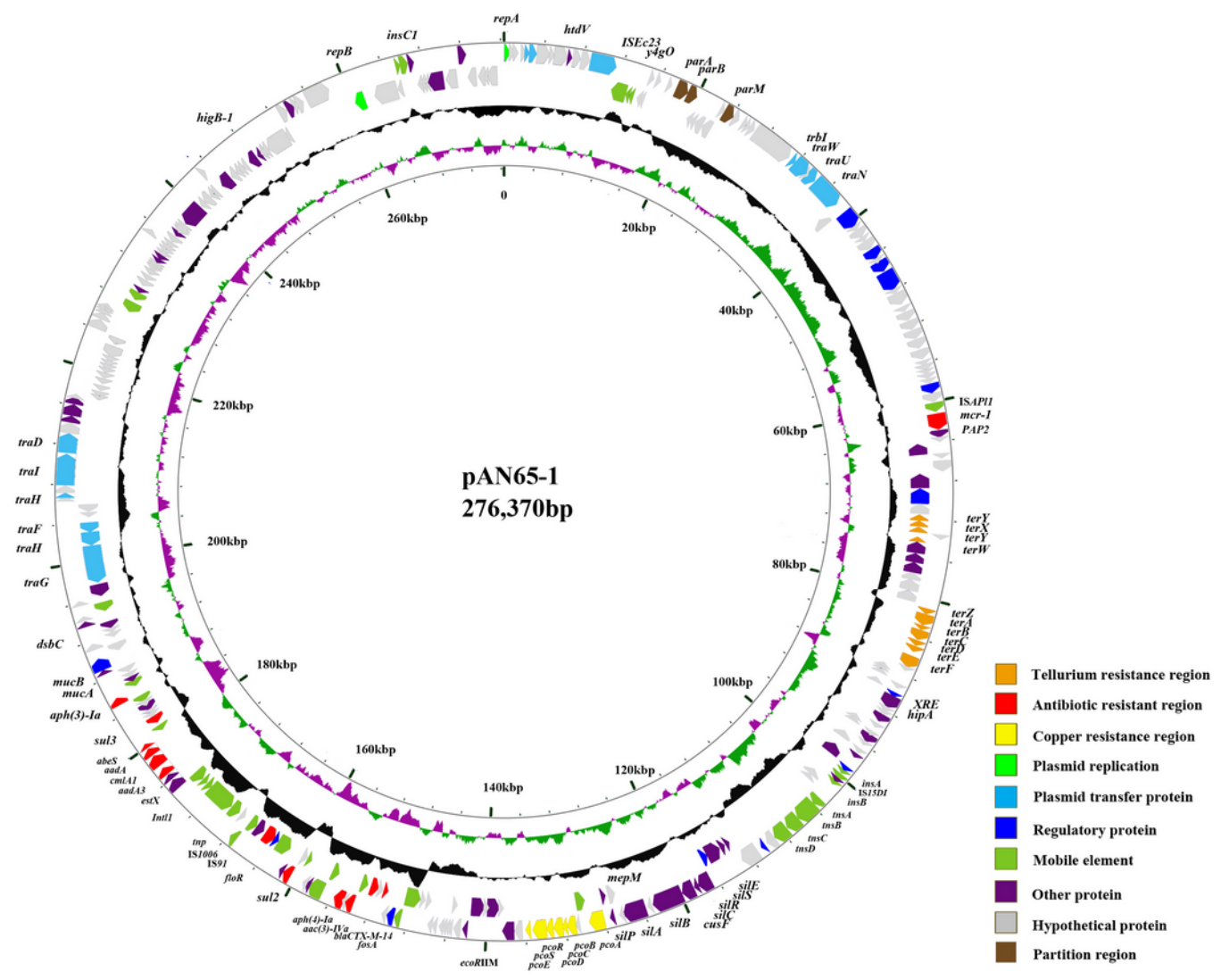

Figure 1

A caption was omitted by the authors in this version of the paper. 


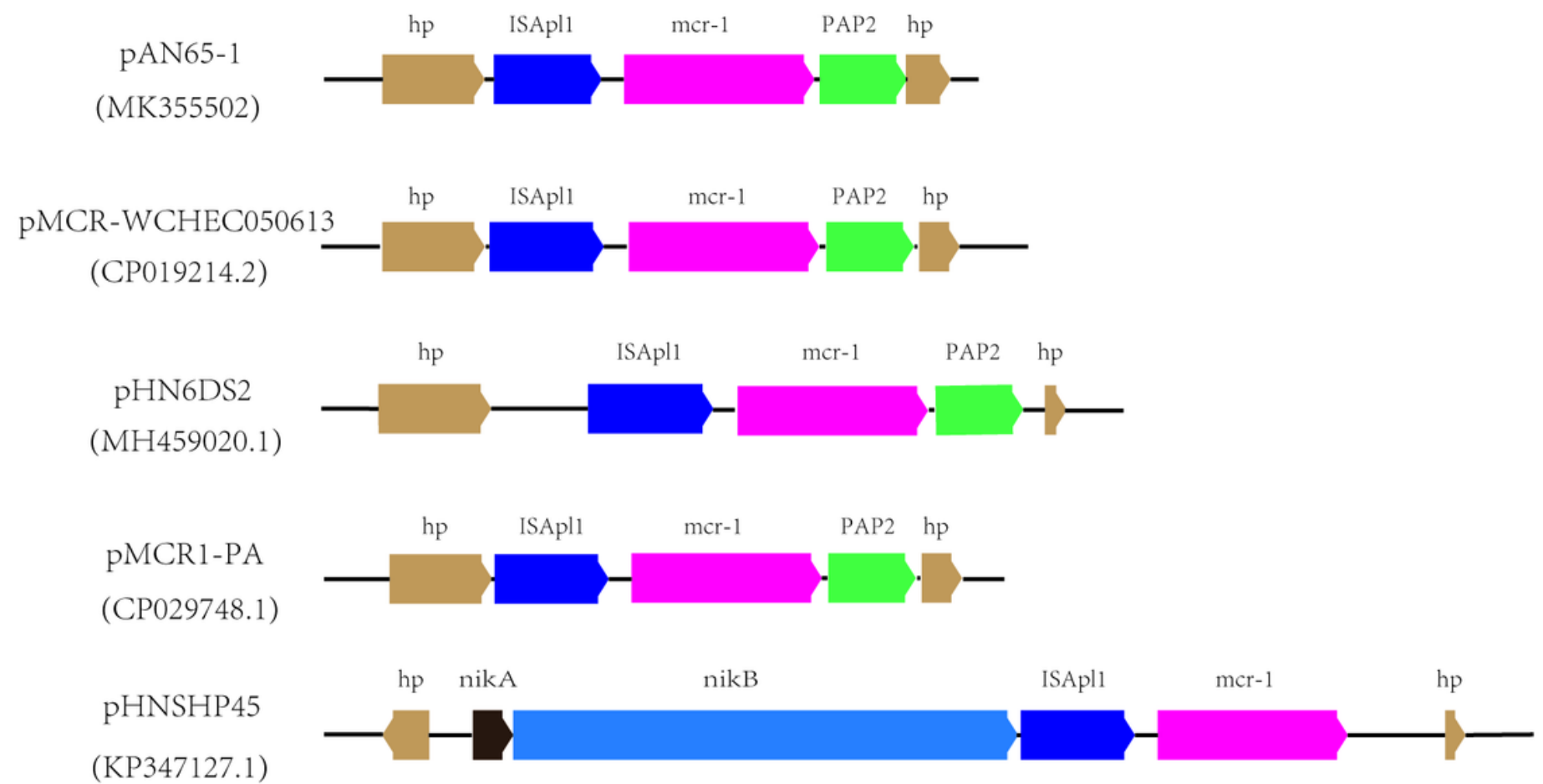

Figure 2

A caption was omitted by the authors in this version of the paper. 


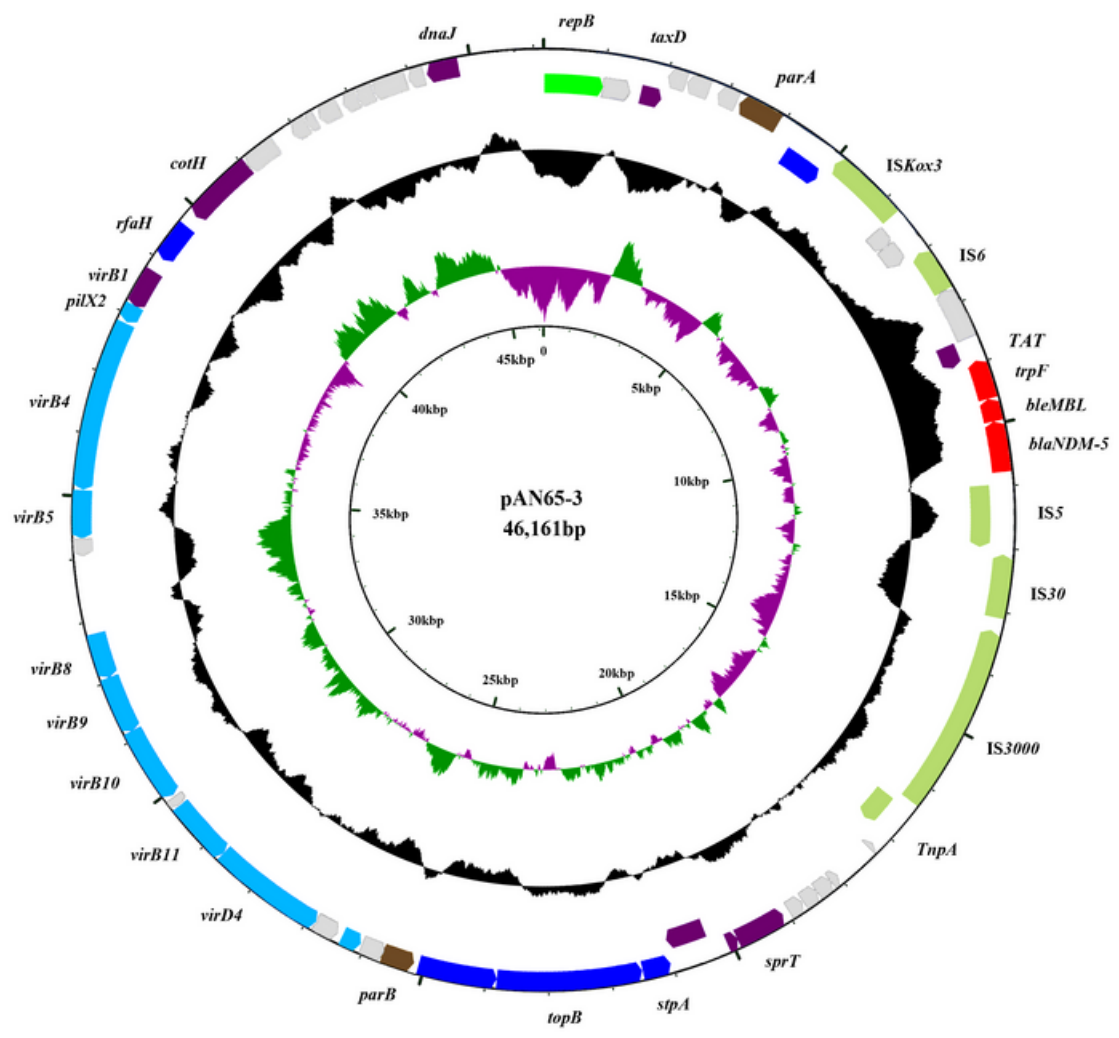

Antibiotic resistant region

Plasmid replication

Plasmid transfer protein

Regulatory protein

Mobile element

Other protein

Hypothetical protein

Partition region

\section{Figure 3}

A caption was omitted by the authors in this version of the paper.

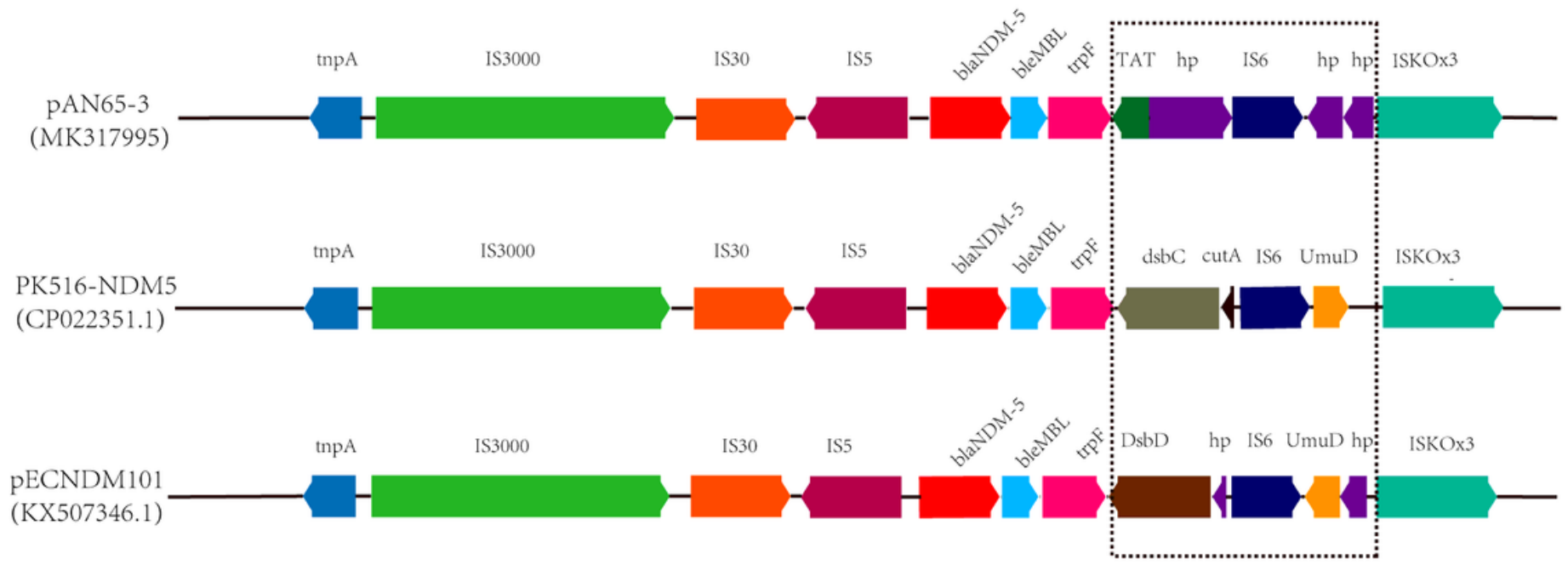

Figure 4

A caption was omitted by the authors in this version of the paper.

\section{Supplementary Files}


This is a list of supplementary files associated with this preprint. Click to download.

- SupplementaryFigure1.tif

- SupplementaryFigure2.PNG

- SupplementaryTable1.doc

- SupplementaryTable2.doc 